\title{
Orbital Angular Momentum on the Light-Front and QCD Observables
}

\author{
Stanley J. Brodsky \\ Stanford Linear Accelerator Center, Stanford University \\ Stanford, California 94309, U.S.A.
}

The light-front wavefunction formalism provides a physical, but rigorous, representation for angular momentum in a relativistic quantum field theory. Each $n$-particle LFWF $\psi_{n}\left(x_{i}, \vec{k}_{\perp} i, S_{i}^{z}\right)$ in the Fock state expansion of a hadron in QCD is frame-independent and satisfies angular momentum conservation $J^{z}=\sum_{i=1}^{n} S_{i}^{z}+\sum_{i=1}^{n-1} L_{i}^{z}$, summed over the $n-1$ independent intrinsic orbital angular momenta $L_{i}^{z}=$ $-i\left[\vec{k}_{i}^{x} \frac{\partial}{\partial \vec{k}_{i}^{y}}-\vec{k}_{i}^{y} \frac{\partial}{\partial \vec{k}_{i}^{x}}\right]$. Gluons propagate with physical polarization $S_{g}^{z}= \pm 1$ in light-cone gauge $A^{+}=0$. All of these features are illustrated by the Fock state expansion of the electron in terms of its fermionboson components.

The light-front formalism provides a representation of hadron physics at the amplitude level. Quark and gluon distributions are computed from the square of the LFWFs and obey DGLAP evolution. The gauge-independent hadron distribution amplitudes $\phi\left(x_{i}, Q\right)$ which control hard exclusive processes is an integral $\int d^{2} k_{\perp} \theta\left(Q^{2}-k_{\perp}^{2}\right) \psi\left(x, \vec{k}_{\perp}\right)$ of the valence LFWF and obeys ERBL evolution. Current matrix elements, and thus all form factors, have an exact representation as overlap of the LFWFs. The Pauli form factor is the matrix element of the $J^{+}$current with opposite $J^{z}$ and thus is nonzero only between states which have $\Delta L_{z}= \pm 1$. In particular, the anomalous magnetic moment is the matrix element of the ladder operator $\vec{J}^{ \pm} \cdot \vec{L}^{\mp}$. Thus, as shown by Drell and myself, the anomalous magnetic moment of any system is nonzero only if the LF Fock expansion contains states with nonzero orbital angular momentum. A similar result holds for the $E$ spin-flip generalized parton distribution. The single-spin asymmetries (Sivers effect) in deep inelastic scattering (and the Drell-Yan process) depend on the same matrix element which enters the Pauli form factor as well as the difference of phases of the final- (initial-) state interactions for Fock states differing by $\Delta L_{z}= \pm 1$. The anomalous gravitomagnetic moment $B(0)$, the spin-flip matrix element of the energy momentum tensor, is found to be zero, Fock state by Fock state, consistent with the equivalence theorem. The electric dipole moment of a hadron is related to the anomalous moment by a factor $\tan \beta$, where $\beta$ is the CP-violating phase of the LFWF.

The AdS/CFT formalism provides a remarkable framework for hadron physics in which conformal symmetry is taken as an initial approximation. Color confinement is introduced by imposing Dirichlet boundary conditions $\phi\left(z=\frac{1}{\Lambda_{Q C D}}\right)=0$ for the hadron amplitude in the fifth dimension. De Teramond and I have found an exact mapping for current matrix elements in AdS space to the corresponding DrellYan West formula for hadron form factors in the light-front formalism. This correspondence leads in turn leads to an exact identification of the $z$ coordinate in AdS/CFT to a variable $\zeta$ in physical spacetime which represents the measure of transverse separation of the constituents within the hadrons. In addition, we have derived effective four-dimensional LF Schrödinger equations for the bound states of massless quarks and gluons which exactly reproduce the AdS/CFT results and give a realistic description of the light-quark meson and baryon spectrum for all orbital angular momentum excitations $L$, as well as form factors for spacelike $Q^{2}$. Only one parameter $\Lambda_{Q C D}$, which sets the mass scale, is introduced.

I also discuss a number of tests of orbital angular momentum in exclusive and inclusive reactions.

This research was supported by the Department of Energy contract DE-AC02-76SF00515. 


$$
\begin{gathered}
\sum_{i}^{n} x_{i}=1 \\
\sum_{i=1}^{n} k_{i}^{+}=\sum_{i=1}^{n} x_{i} \vec{P}^{+}=\vec{P}^{+} \\
\Psi_{n}\left(x_{i}, \vec{k}_{\perp i}, \lambda_{\perp i}\right) \overrightarrow{0}_{\perp} \\
\sum_{i=1}^{n}\left(x_{i} \vec{P}_{\perp}+\vec{k}_{\perp i}\right)=\vec{P}_{\perp} \\
\vec{\ell}_{j} \equiv\left(\vec{P}_{\perp} \times \vec{b}_{\perp}\right)_{j}=\left(\vec{k}_{\perp} \times \frac{i \partial}{\partial \vec{k}_{\perp}}\right)_{j} \\
\text { n-I Intrinsic Orbital Angular Momenta } \\
\text { Frame Independent } \quad j=1,2, \cdots(n-1)
\end{gathered}
$$

New Mexico $2-24-06$
Orbital Angular Momentum in QCD
Stan Brodsky, SLAC 


$$
\frac{F_{2}\left(q^{2}\right)}{2 M}=\sum_{a} \int[\mathrm{d} x]\left[\mathrm{d}^{2} \mathbf{k}_{\perp}\right] \sum_{j} e_{j} \frac{1}{2} \times
$$

$\left[-\frac{1}{q^{L}} \psi_{a}^{\uparrow *}\left(x_{i}, \mathbf{k}_{\perp i}^{\prime}, \lambda_{i}\right) \psi_{a}^{\downarrow}\left(x_{i}, \mathbf{k}_{\perp i}, \lambda_{i}\right)+\frac{1}{q^{R}} \psi_{a}^{\downarrow *}\left(x_{i}, \mathbf{k}_{\perp i}^{\prime}, \lambda_{i}\right) \psi_{a}^{\uparrow}\left(x_{i}, \mathbf{k}_{\perp i}, \lambda_{i}\right)\right]$

$$
\mathbf{k}_{\perp i}^{\prime}=\mathbf{k}_{\perp i}-x_{i} \mathbf{q}_{\perp} \quad \mathbf{k}_{\perp j}^{\prime}=\mathbf{k}_{\perp j}+\left(1-x_{j}\right) \mathbf{q}_{\perp}
$$

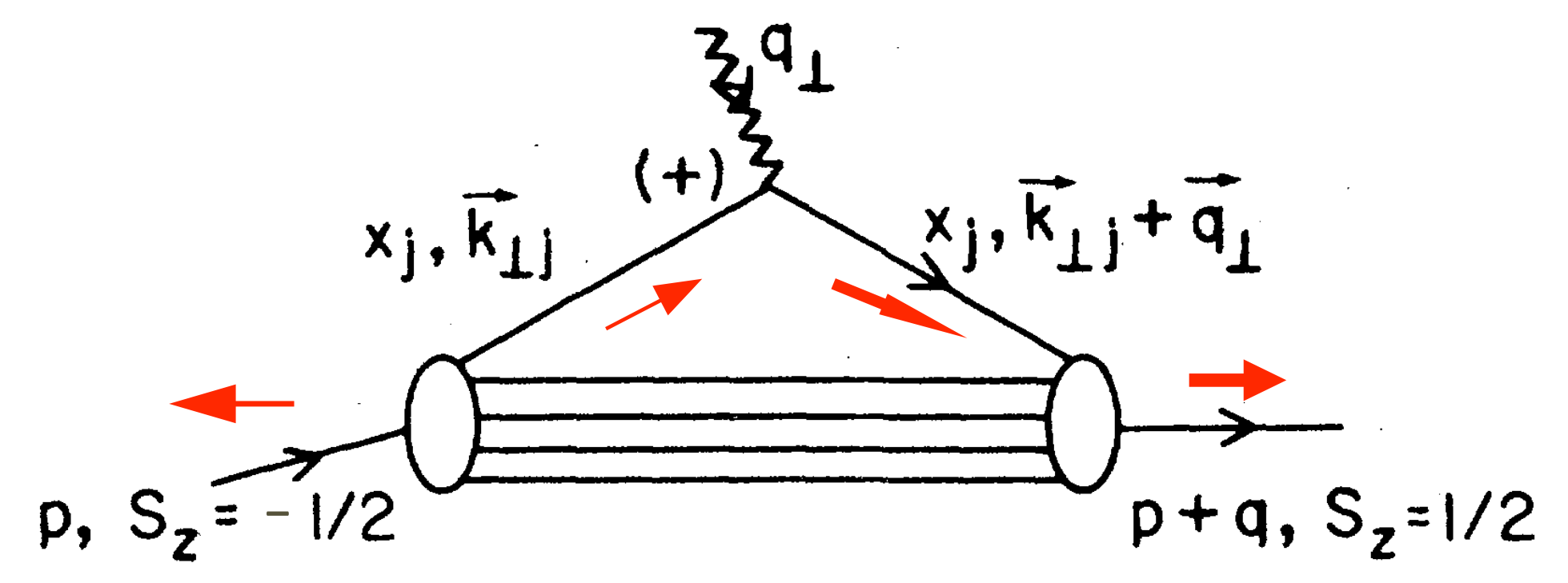

Must have $\Delta \ell_{z}= \pm 1$ to have nonzero $F_{2}\left(q^{2}\right)$

New Mexico

$2-24-06$
Orbital Angular Momentum in QCD

35
Stan Brodsky, SLAC 


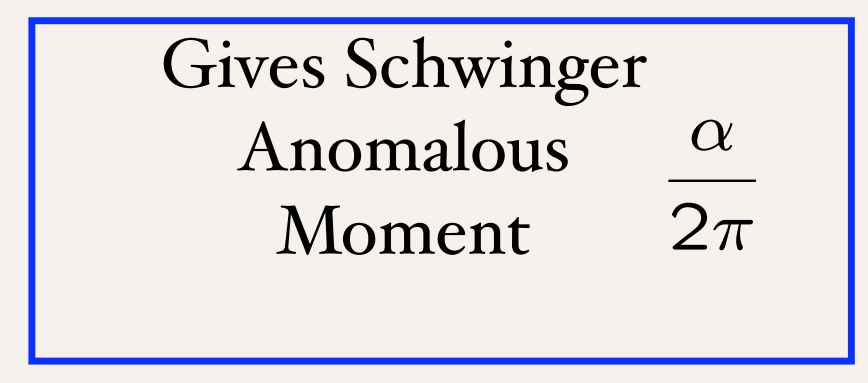

$$
\begin{cases}\psi_{+\frac{1}{2}+1}^{\uparrow}\left(x, \vec{k}_{\perp}\right)=-\sqrt{2} \frac{\left(-\mathrm{k}^{1}+\mathrm{i} \mathrm{k}^{2} \mid\right.}{\mathrm{x}|1-\mathrm{x}|} \varphi, & L_{z}=-1 \\ \psi_{+\frac{1}{2}-1}^{\uparrow}\left(x, \vec{k}_{\perp}\right)=-\sqrt{2} \frac{\left.\left|+\mathrm{k}^{1}+\right| \mathrm{k}^{2}\right)}{1-\mathrm{x}} \varphi, & L_{z}=1 \\ \psi_{-\frac{1}{2}+1}^{\uparrow}\left(x, \vec{k}_{\perp}\right)=-\sqrt{2}\left(M-\frac{\mathrm{m}}{\mathrm{x}}\right) \varphi, & L_{z}=0 \\ \psi_{-\frac{1}{2}-1}^{\uparrow}\left(x, \vec{k}_{\perp}\right)=0 & \end{cases}
$$

where

$$
\varphi=\varphi\left(x, \vec{k}_{\perp}\right)=\frac{e / \sqrt{1-x}}{M^{2}-\left(\vec{k}_{\perp}^{2}+m^{2}\right) / x-\left(\vec{k}_{\perp}^{2}+\lambda^{2}\right) /(1-x)}
$$

$$
M \rightarrow \mathrm{m}+\lambda^{l}
$$

Spin-I mass $\lambda^{l}$

Spin-I/2 mass m

$$
\left\{\begin{array}{l}
\psi_{+\frac{1}{2}+1}^{\downarrow}\left(x, \vec{k}_{\perp}\right)=0 \\
\psi_{+\frac{1}{2}-1}^{\downarrow}\left(x, \vec{k}_{\perp}\right)=-\sqrt{2}\left(M-\frac{\mathrm{m}}{\mathrm{x}}\right) \varphi \\
\psi_{-\frac{1}{2}+1}^{\downarrow}\left(x, \vec{k}_{\perp}\right)=-\sqrt{2} \frac{\left(-\mathrm{k}^{1}+\mathrm{ik}\right)}{1-\mathrm{x}} \varphi \\
\psi_{-\frac{1}{2}-1}^{\downarrow}\left(x, \vec{k}_{\perp}\right)=-\sqrt{2} \frac{\left(+\mathrm{k}^{1}+\mathrm{k}^{2}\right)}{\mathrm{x}(1-\mathrm{x})} \varphi
\end{array}\right.
$$

Drell, sjb Hwang, Schmidt, sjb
New Mexico $2-24-06$
Orbital Angular Momentum in QCD
Stan Brodsky, SLAC 


\section{Measure Orbital Angular Momentum in Diffractive Reactions}

$\bullet$ $p A \rightarrow q q q A \quad$ Coulomb Excitation $p e \rightarrow q q q e$

- Test Color Transparency

- Measure $\psi_{p}\left(x_{i}, \vec{k}_{\perp i}, \lambda_{i}\right)$

- Angular distributions reveal $S$ vs. $P$ waves

- Correlations with Spin of Projectile Proton

$\gamma^{*} p \rightarrow q \bar{q} p$

New Mexico 2-24-06 


\section{Orbital Angular Momentum and Hard Exclusive Reactions}

- LFWFs with L nonzero have faster fall-off at short distances

$$
\psi\left(x, \vec{k}_{\perp}\right) \rightarrow\left[\frac{\left|\vec{k}_{\perp}\right|}{\sqrt{x(1-x)}}\right]^{L}\left[\frac{x(1-x)}{\vec{k}_{\perp}^{2}}\right]^{1+L},
$$

- Faster fall-off of hard exclusive reactions

- Hadron helicity conservation violation

$$
\frac{u_{\downarrow}(x)}{u_{\uparrow}(x)} \sim(1-x)^{2}
$$

- Spectator rule for structure functions at $x \rightarrow 1$

- Spectacular violations, spin anomalies

- Ann in p p scattering

- $J / \psi \rightarrow \rho \pi$ puzzle 


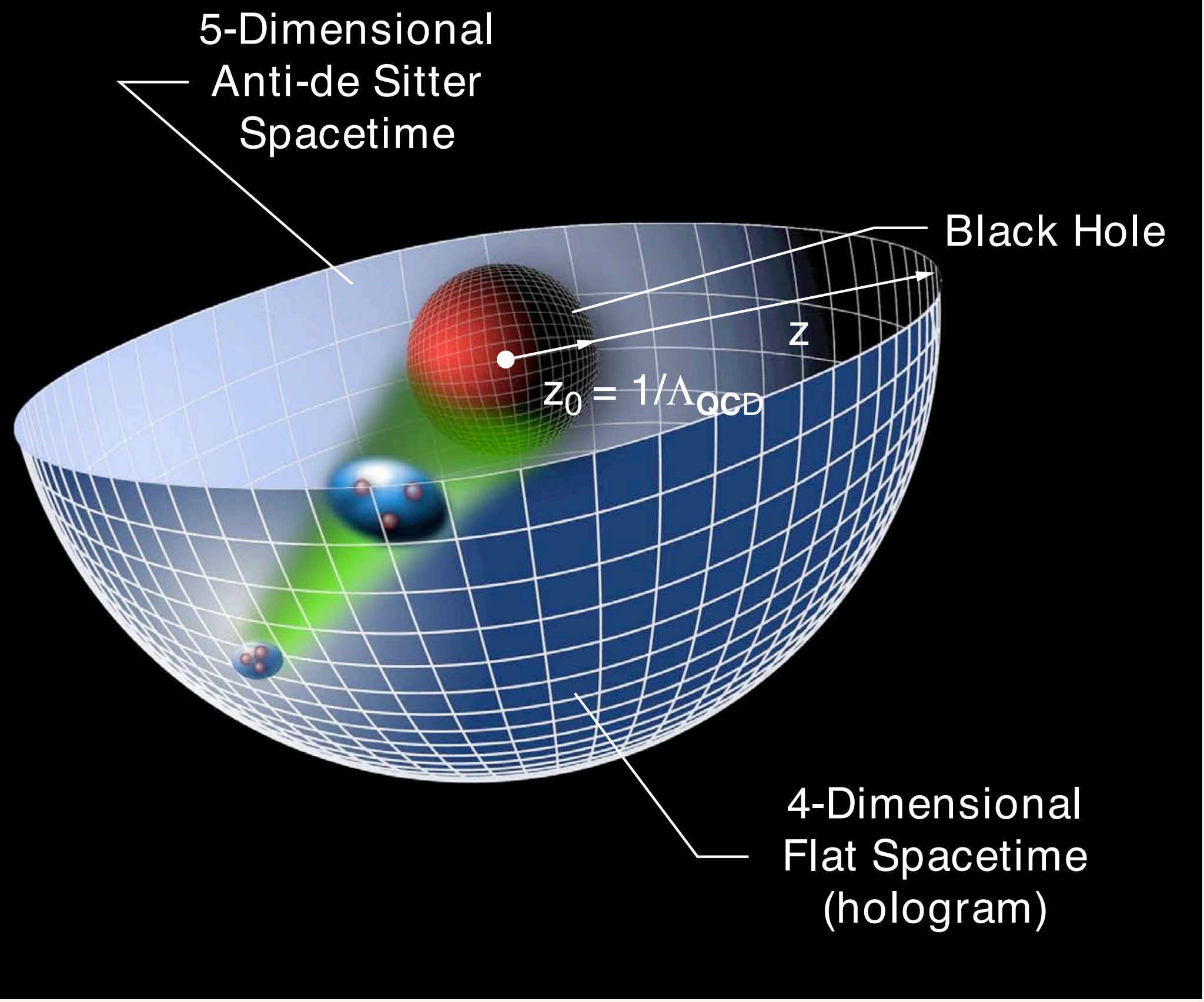

New Mexico $2-24-06$
Orbital Angular Momentum in QCD

92
Stan Brodsky, SLAC 
- Pseudoscalar mesons: $\mathcal{O}_{3+L}=\bar{\psi} \gamma_{5} D_{\left\{\ell_{1}\right.} \ldots D_{\left.\ell_{m}\right\}} \psi \quad\left(\Phi_{\mu}=0\right.$ gauge $)$.

- 4- $d$ mass spectrum from boundary conditions on the normalizable string modes at $z=z_{0}$, $\Phi\left(x, z_{o}\right)=0$, given by the zeros of Bessel functions $\beta_{\alpha, k}: \mathcal{M}_{\alpha, k}=\beta_{\alpha, k} \Lambda_{Q C D}$

- Normalizable AdS modes $\Phi(z)$

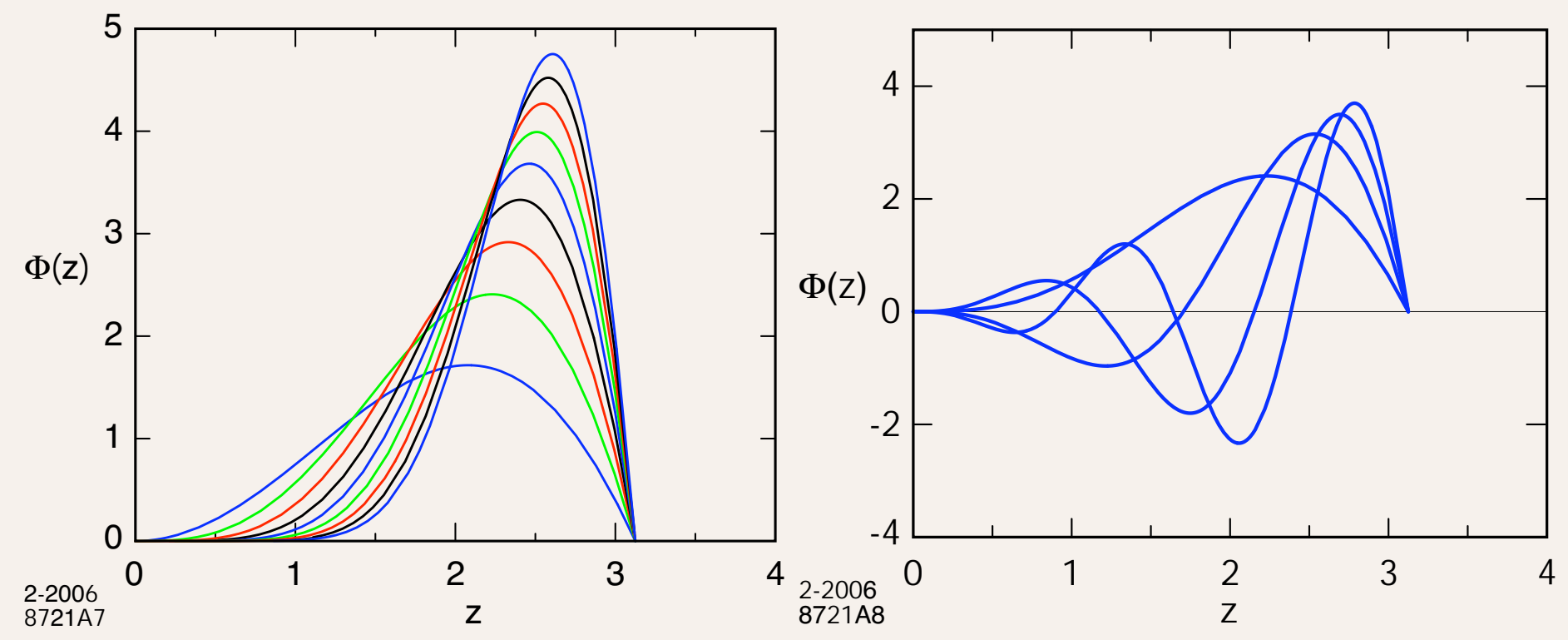

Fig: Meson orbital and radial AdS modes for $\Lambda_{Q C D}=0.32 \mathrm{GeV}$.

New Mexico $2-24-06$
Orbital Angular Momentum in QCD 99
Stan Brodsky, SLAC 
(a)

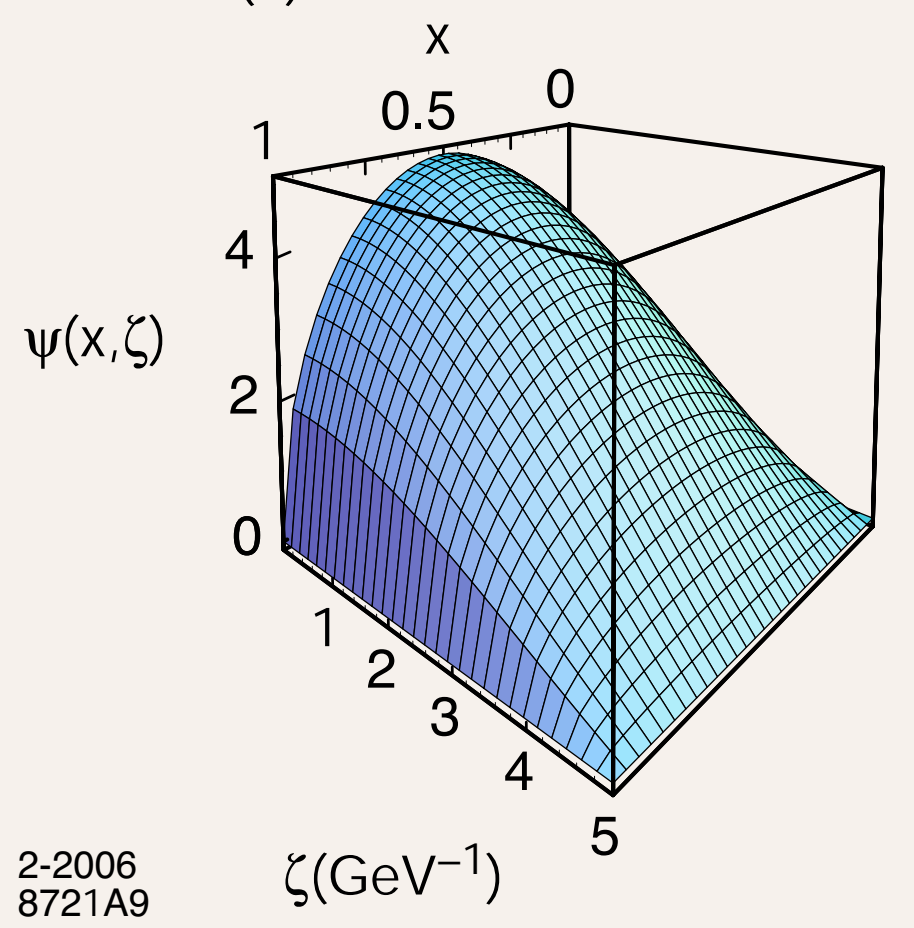

(b)

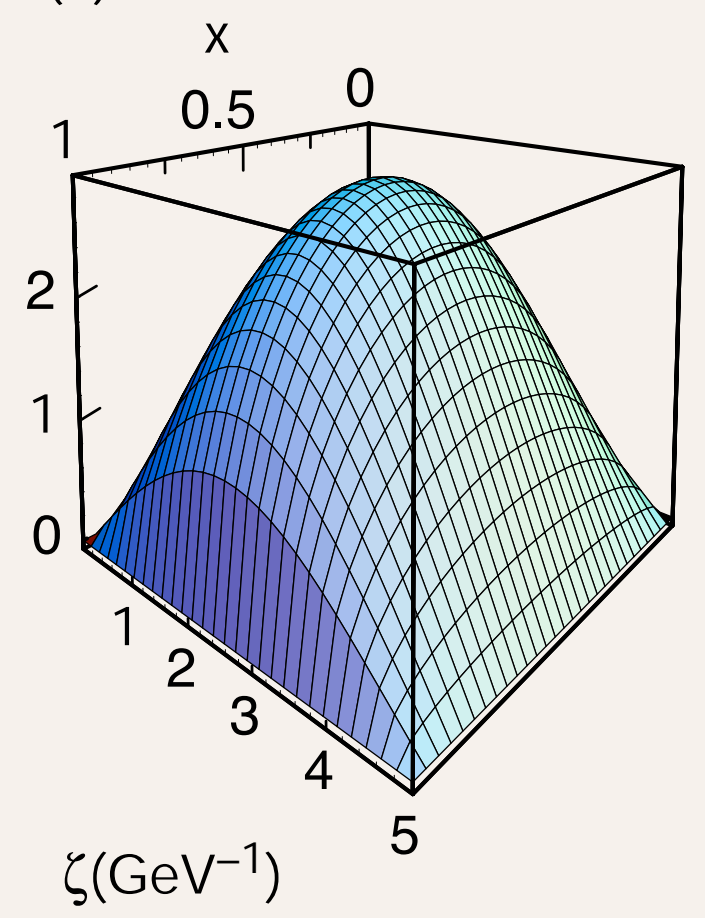

(c)

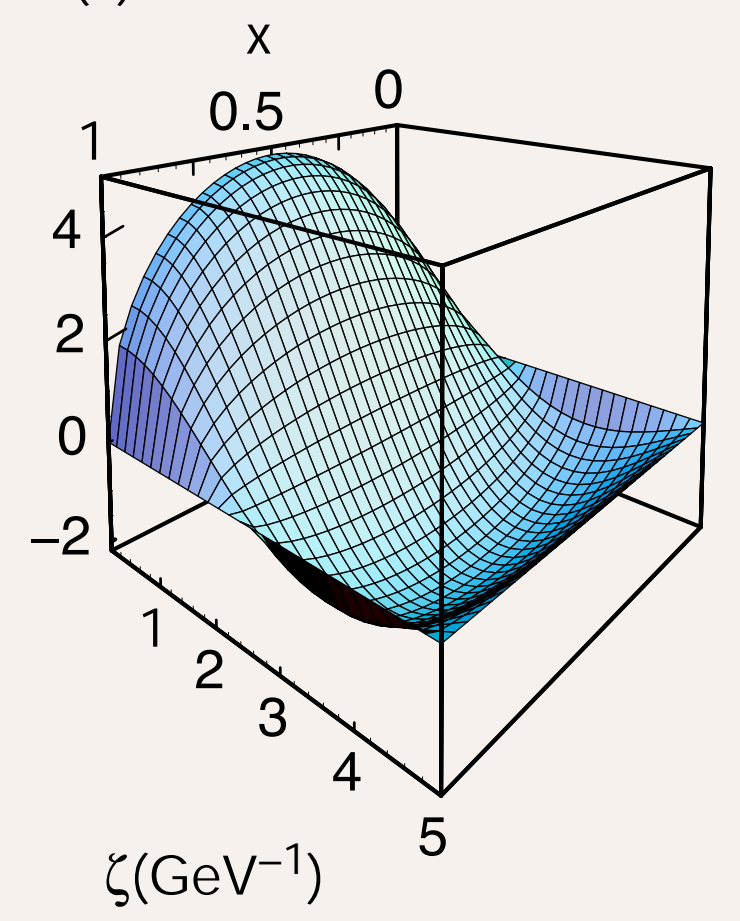

Two-quark holographic LFWF in impact space $\psi(x, \zeta):(a) \ell=0, k=1 ;(b) \ell=1, k=1$; c) $\ell=0, k=2$. The variable $\zeta$ is the holographic variable $z=\zeta=\left|b_{\perp}\right| \sqrt{x(1-x)}$.

New Mexico $2-24-06$
Orbital Angular Momentum in QCD

IO2
Stan Brodsky, SLAC 


\section{Map AdS/CFT to $3^{+1}$ LF Theory}

Effective radial equation:

$$
\left[\begin{array}{r}
\left.-\frac{d^{2}}{d \zeta^{2}}+V(\zeta)\right] \phi(\zeta)=\mathcal{M}^{2} \phi(\zeta) \\
\zeta^{2}=x(1-x) \mathbf{b}_{\perp}^{2}
\end{array}\right.
$$

Effective conformal potential:

$$
V(\zeta)=-\frac{1-4 L^{2}}{4 \zeta^{2}} .
$$

G. de Teramond and sjb

General solution:

\section{(preliminary)}

$$
\begin{aligned}
& \widetilde{\psi}_{L, k}\left(x, \vec{b}_{\perp}\right)=B_{L, k} \sqrt{x(1-x)} \\
& J_{L}\left(\sqrt{x(1-x)}\left|\vec{b}_{\perp}\right| \beta_{L, k} \Lambda_{\mathrm{QCD}}\right) \theta\left(\vec{b}_{\perp}^{2} \leq \frac{\Lambda_{\mathrm{QCD}}^{-2}}{x(1-x)}\right),
\end{aligned}
$$

New Mexico $2-24-06$
Orbital Angular Momentum in QCD II 7
Stan Brodsky, SLAC 
- Only one scale $\Lambda_{Q C D}$ determines hadronic spectrum (slightly different for mesons and baryons)

- Light-cone frame is the natural frame to establish the AdS/QCD holographic duality.

- Ratio of Nucleon to Delta trajectories determined by zeroes of Bessel functions.

- AdS modes dual to hadrons extrapolate to valence constituents at zero separation in the AdS boundary.

- Non-zero orbital angular momentum and higher Fock-states require introduction of quantum fluctuations.

- Initial good approximation for description of the structure of hadronic form factors and other observables.

- Use of holographic light-front wave functions to compute hadronic matrix elements.

- Dominance of quark-interchange in hard exclusive processes emerges naturally from the classical duality of the holographic model, modified by gluonic quantum fluctuations.

- Covariant version of the bag model with confinement and conformal symmetry.

- Precise mapping of string modes to partonic states. Modes inside AdS represent the probability amplitude for the distribution of quarks at a given scale.

- Exact holographic mapping for $n$-parton state determines effective QCD transverse charge density in terms of modes in AdS space.

- Holographic mapping allows deconstruction: express the eigenvalue problem in terms of 3+1 QCD degrees of freedom. 


\section{Hadron Dynamics at the Amplitude Level}

- LFWFS are the universal hadronic amplitudes that underlie structure functions, GPDs, exclusive processes.

- Relation of transversity and other distributions to physics of the hadron itself.

- Connections between observables

- GPDs are not densities or probability distributions

- Parton number not conserved: n=n' \& n=n'+2 at nonzero skewness

- orbital angular momentum

- Role of FSI and ISIs--Sivers effect

New Mexico $2^{-24}-06$
Orbital Angular Momentum in QCD I23
Stan Brodsky, SLAC 